\title{
REVIEW
}

\section{Glycocalyx in Endotoxemia and Sepsis}

\author{
Michael S. Goligorsky* ${ }^{\star \ddagger}$ and Dong Sun
}

From the Departments of Medicine, ${ }^{*}$ Pharmacology, ${ }^{\dagger}$ and Physiology, ${ }^{\ddagger}$ New York Medical College, Valhalla, New York

Accepted for publication
June 6, 2019.
Address correspondence to
Michael S. Goligorsky, M.D.,
Ph.D., New York Medical Col-
lege, 15 Dana Rd., Basic Sci-
ences Bldg., Valhalla, NY
10595. E-mail: michael_-
goligorsky@nymc.edu.

Accepted for publication

6,2019

Michael S. Goligorsky, M.D., Ph.D., New York Medical Colege, 15 Dana Rd., Basic Scices Bldg., Valhalla, NY 595. E-mail: michael

Sepsis is an ominous clinical manifestation of a severe generalized infection leading to systemic multiorgan sequelae such as hypotension, acute respiratory distress syndrome, renal failure, and lactic acidosis, among others. It afflicts $>750,000$ people each year in the United States alone and has a mortality rate of $28 \%$ to $50 \%$. One of the key molecular causes of gram-negative septicemia is a component of the outer membrane of gram-negative bacteria, lipopolysaccharide (LPS). It binds with high affinity to LPS-binding glycoproteins and activates Toll-like receptor (TLR)-4 and co-receptor CD14 expressed on monocytes/ macrophages and endothelial cells to induce the secretion of proinflammatory cytokines. ${ }^{1}$ This is, however, not the only and, perhaps, not the main pathogenic mechanism of LPS. Recent clinical trials of TLR4 inhibitors have not produced an expected amelioration of severe sepsis. ${ }^{2,3}$ An alternative intracellular LPS-sensing pathway, bypassing TLR4, that activates caspases 4 and 5 in humans and 11 in mice and resulting in inflammasome-3-induced pyroptosis has been considered to be the main pathogenic mechanism of sepsis. ${ }^{4,5}$

The incidence of sepsis is on a rise, while the mortality rate maintains the level of about $30 \%$. $^{6}$ With the conceptual shift from the idea of a "disproportionate", cytokine response to infection in septic shock to that of a "severe endothelial dysfunction syndrome in response to intravascular and extravascular infection", pp.1743 came the emphasis on the crucial role of impaired microcirculation to vital organs as the leading mechanism of sepsis and septic shock.

Considering the systemic nature of septicemia, vascular endothelium represents the front line of exposure to bacterial endotoxins. Endothelial dysfunction and impaired microcirculation develop in the course of sepsis and determine its severity and duration ${ }^{8,9}$ This role of microcirculation and microvascular endothelium in the pathogenesis of septic multiorgan failure has been known for decades, ${ }^{1,10-12}$ but recent studies have implicated endothelial glycocalyx (EG) in at least some manifestations of endothelial dysfunction in sepsis. ${ }^{10-13} \mathrm{We}$ and others have shown that preventive strategies for reducing the degradation of EG result in improved survival in mice with polymicrobial sepsis. $^{10,13,14}$ The global loss of EG in polymicrobial sepsis has been documented by Song et $\mathrm{al}^{14}$ using dilution

Supported by NIH grant HL144528 (M.S.G. and D.S.).

Disclosures: None declared.

This article is part of a review series on glycocalyx in human disease. No person at New York Medical College was involved in the peer review process or final disposition of this article. 
technique with two fluorophores, glycocalyx-penetrating and -nonpenetrating probes. All of these studies have prompted the present focus on EG in sepsis.

EG covers most of the vascular luminal surface. Twophoton laser scanning microscopy of murine carotid arteries labeled with fluorescent wheat germ agglutinin and visualized in areas prone and not prone to develop atherosclerotic plaques showed labeling of $>90 \%$ of the surface in nonprone areas of the common carotid artery, with only $73 \%$ of the surface area in atherogenesis-prone internal carotid arteries ${ }^{15}$ and the thickness of glycocalyx reaching 2.3 to 2.5 $\mu \mathrm{m}$. These findings are in part supported by the results obtained using high-resolution confocal microscopy and labeling of glycocalyx with antibodies against heparan sulfate (HS), ${ }^{16}$ which revealed the thickness of glycocalyx varying between 0.9 and $1.5 \mu \mathrm{m}$. Of note, in this study the access to the glycocalyx for ex vivo perfused wheat germ agglutinin-fluorescein isothiocyanate, which binds to sialic acid and $N$-acetylglucosaminyl residues, was impeded, and labeling required prior perfusion of an air bubble, allegedly improving the access of the fluoroprobe to its targets in the glycocalyx.

\section{Vicious Circle of Glycocalyx Degradation and Endothelial Dysfunction}

As mentioned above, EG is an early victim of endotoxemia; so is the function of endothelial cells. ${ }^{17}$ The question is: Are those two events related? We proposed the concept of a vicious circle-type relationship between endothelial dysfunction and the loss of glycocalyx. This concept, stemming from a score of previous studies, ${ }^{8}$ has been formulated to explain the tight association between the two conditions and to emphasize the need for therapeutic interventions targeting both, as summarized by Zhang et al. ${ }^{18}$ Endothelial cell dysfunction-as characterized by reduced nitric oxide (NO) bioavailability, enhanced production of reactive oxygen species, activation of sheddases, and impaired exocytosis of lysosome-related organelles (Lysosome-Related Organelles) - triggers degradation of EG, which, in turn, leads to defective shear stress-induced activation of endothelial NO synthase and further decline in NO bioavailability, shedding of components of glycocalyx endowed with proinflammatory properties, and loss of glycocalyx-bound growth and survival factors, thus fueling the vicious circle.

\section{Metabolic Signatures of Sepsis as Related to Glycocalyx}

In general, sepsis is characterized as a catabolic state of degradation of proteins, lipids, and carbohydrates. ${ }^{19}$ Remarkably, recent advanced metabolic gene expression systems analysis of LPS-induced endothelial dysfunction revealed an increase in glycan production (together with fatty acid metabolism) to accompany glycocalyx loss in endothelial cells exposed to LPS and an increase in their permeability by $60 \%{ }^{20}$ Confirmation of these in vitro findings in patients with sepsis showed that upregulation of glycan synthesis was among the worst-affected areas of metabolism, being most deranged in nonsurvivors. The data are consistent with a view of compensatory induction of synthesis of structural components of glycocalyx, among others, upon application of glycocalyxdegrading stressors such as LPS, thus suggesting a tight feedback control mechanism coupling its synthesis and degradation. The fact that the degradation of glycocalyx in sepsis prevails over increased synthesis of at least one of its components strongly favors its enhanced enzymatic degradation. It is carried out by sheddases, a disintegrin and metalloproteinases (ADAMs), matrix metalloproteinase (MMPs), heparanase-1, and hyaluronidases (Figure 1).

\section{Mechanisms of Glycocalyx Degradation}

\section{ADAM17, ADAM10, and MMPs}

ADAMs show sequence similarity, yet ADAM10 is expressed constitutively, while ADAM17 is inducible, as it is converted into an active form upon cell stimulation. ${ }^{21}$ In addition to the well-known conversion of tumor necrosis factor (TNF)- $\alpha$ to the soluble form, ADAM17 cleaves syndecan-4, whereas ADAM10 sheds syndecan-1. Among other ADAM targets are receptors for IL- 6 , TNF, and cell adhesion molecules ICAM-1, VCAM-1, L-selectin. All of those receptors are expressed by leukocytes and endothelial cells. ADAM17 activity is induced in sepsis, thus resulting in shedding components of leukocyte-endothelial cell tether machinery and facilitating systemic inflammation. This shedding explains the immune paralysis and impaired microbial clearance in patients with sepsis. In contrast to ADAM17, ADAM13 activity is suppressed in sepsis, ${ }^{22}$ thus compromising cleavage of von Willebrand factor multimers and predisposing to the development of intravascular platelet aggregation and thrombosis. Members of the ADAMs family and MMPs (a.k.a. matrixins) are zinc-dependent endopeptidases sharing many structure-functional properties, as well as substrates-extracellular matrix proteins and bound to them growth factors (insulin-like growth factor, vascular endothelial growth factor, transforming growth factor $\beta$, fibroblast growth factor, and others), cytokines and chemokines. In addition, they can activate $\mathrm{TNF} \alpha$ and alter CD44-hyaluronic acid (HA) interaction. ${ }^{23}$ MMPs are induced in sepsis, leading to impairment of host defense. ${ }^{24}$ While the search for highly specific and selective inhibitors is proceeding, it is noteworthy that systemic application of MMP inhibitors may have a broad spectrum of untoward effects, potentially limiting their clinical utility.

\section{Heparanase}

Heparanase gene expression is epigenetically and p53 controlled, but can be stimulated by early growth response 1 


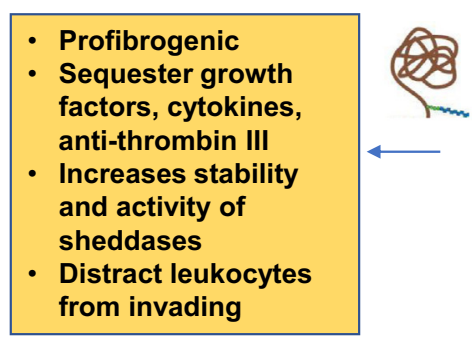

Proteoglycan core

protein ectodomains

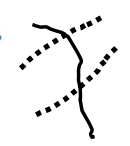

(
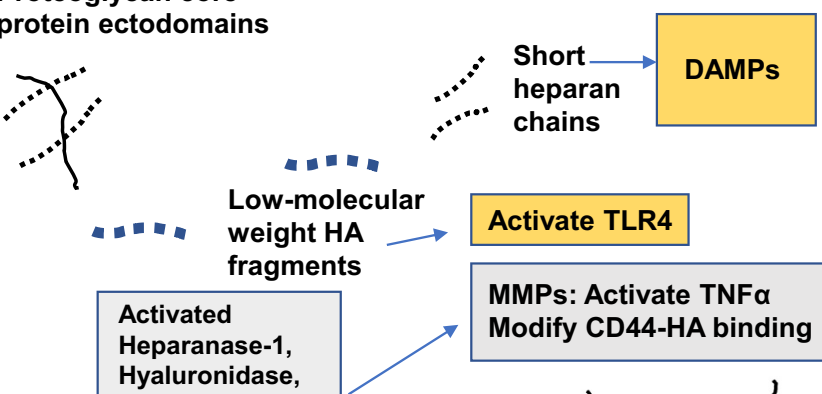

MMPs: Activate TNFa Modify CD44-HA binding

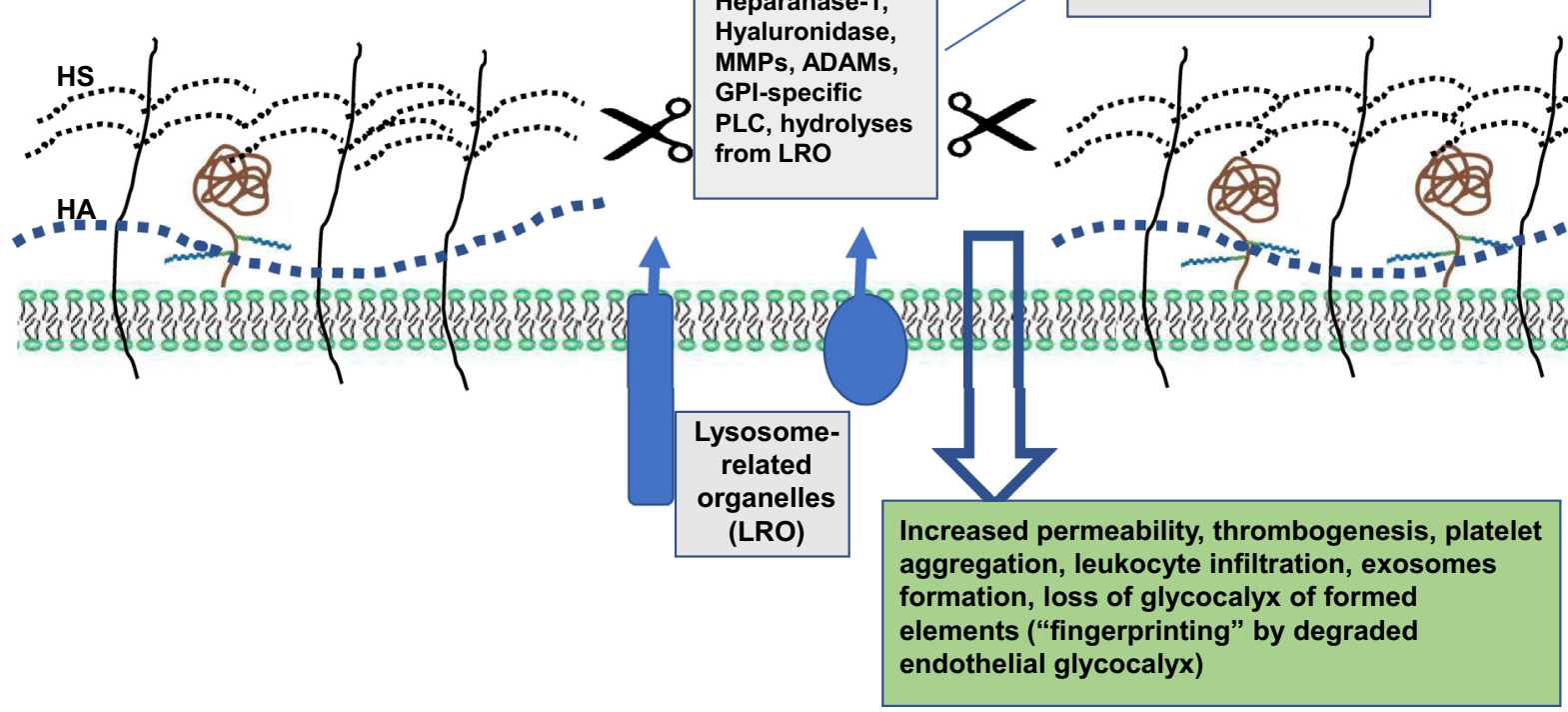

Figure 1 Mechanisms of glycocalyx degradation, pathogenic role of liberated fragments of glycocalyx and pathophysiological consequences of the loss of endothelial glycocalyx. The pathways of enzymatic degradation of components of glycocalyx are presented in the central gray boxed areas. Pathogenic features of released fragments of proteoglycan core proteins, short heparan chains, and low-molecular-weight hyaluronic acid (HA) are depicted as yellow boxed areas. The consequences of endothelial glycocalyx degradation are summarized in the green boxed area. ADAM, a disintegrin and metalloproteinase; DAMP, danger-associated molecular patterns; GPI, glycosylphosphatidylinositol; HS, heparan sulfate; MMP, matrix metalloproteinase; PLC, phospholipase C; TNF, tumor necrosis factor; TLR, Toll-like receptor.

transcription factor, reactive oxygen species, and inflammatory cytokines. ${ }^{25}$ Several mutated variants of p53 induce heparanase expression, thus explaining its overexpression in some malignant tumors. ${ }^{26}$ The enzyme is activated in sepsis, with the consequent degradation of HS moieties, further aggravating the loss of glycocalyx components. The pathogenic role of heparinase activation in sepsis-induced respiratory distress has been convincingly demonstrated by Schmidt et $\mathrm{al}^{10}$ through the application of heparinase inhibitors and the use of heparanase-deficient mice. Similar observations were made in a model of ischemic acute kidney injury.

\section{Hyaluronidase}

HA receptors are many and, in addition to CD44, include receptor for HA-mediated motility, lymphatic vessel endothelial receptor 1, HA receptor for endocytosis, and TLR4, thus making HA a potent signaling molecule, with its repertoire being further enriched by the opposing actions of high - and low-molecular-weight species. ${ }^{27,28}$ Hyaluronan metabolism is affected in sepsis. Circulating levels of HA and HS are increased fourfold in patients with sepsis, ${ }^{29}$ being higher in those who did not survive 90 days, but only HA levels were correlated with the severity of kidney and liver functional damage. In a unilateral renal ischemia reperfusion model in rats, HA was risen at day 1, predominantly as a high-molecular-weight species, with later dominance of lower-molecular-weight fragments. This phenomenon is associated with a 35 - to 50 -fold increase in hyaluronan synthase mRNA transcripts in the outer and inner medullary stripes and continuing elevation of hyaluronan synthase 2 mRNA. In parallel, the activities of hyaluronidases 1 and 2 are repressed during the first 24 hours after ischemia reperfusion. ${ }^{30}$

\section{Lysosome-Related Organelles}

Lysosome-related organelles trigger EG degradation. Intriguingly, the luminal surface of lysosomes, as well as late endosomes and autophagosomes, is coated with a thin glycoconjugate-rich layer, referred to as lysosomal glycocalyx. ${ }^{31}$ This layer, enriched in lysosomal-associated membrane proteins 1 and 2, is believed to protect the limiting membrane from autodigestion by diverse hydrolyses contained inside lysosomes. ${ }^{32}$ Electron microscopy and 
computational analysis of the crystal structure of a member of lysosomal-associated membrane proteins posit that the thickness of lysosomal glycocalyx approximates 5 to 12 $\mathrm{nm} .{ }^{31}$ Although it has been suggested that exocytosis of secretory lysosomes may assist in reconstituting plasma membrane glycocalyx, the dimensions of lysosomal glycocalyx are hardly sufficient for this purpose. In fact, we believe that the opposite is occurring under endotoxemic stress. ${ }^{13,33}$ Exocytosis of lysosome-related organelles is detectable within minutes after the application of the stressor. Notably, lysosomes contain nearly 60 different soluble hydrolases, including sulphatases, peptidases, phosphatases, lipases, and nucleases, which, although active in acidic $\mathrm{pH}$, retain nearly $20 \%$ activity at neutral $\mathrm{pH}$ and can hydrolyze glycosaminoglycans, sphingolipids, and an array of proteins. ${ }^{34}$ This singular exocytotic event results in the liberation of a multitude of stored components from the Weibel-Palade bodies and secretory lysosomes. Through the use of stochastic optical reconstruction microscopy of cultured cells stained with anti-HS antibodies, Zullo et al ${ }^{13}$ illustrated the appearance of patchy degradation of EG already 10 to 15 minutes after the application of LPS. This phenomenon was associated with the increased levels of cathepsin B in the culture medium, consistent with the export of lysosomal cargo. When the docking of lysosomerelated organelles was inhibited with NO donor, the loss of EG was mitigated. In addition to heparanase-mediated and mast cell-mediated sepsis-induced degradation of glycocalyx, these findings provide a yet another pathway of early loss of this structure, as depicted in Figure 1. The proposed role for exocytosed lysosomal cargo in degradation of glycocalyx has multiple broad-ranging biological equivalents, such as osteoclastic bone resorption, platelet induction of coagulation, spermatozoan hydrolysis in fertilization, and the defense of mast cells and eosinophils against parasites, to name a few examples. All of those mechanisms of shedding of glycocalyx are compiled in Figure 1.

\section{Clinical Manifestations of Sepsis as Related to Glycocalyx}

\section{Pulmonary Manifestations}

Pulmonary manifestations of sepsis are perilous. Pulmonary microvasculature is one of the critically damaged targets of sepsis, leading to acute respiratory distress syndrome. A study by Schmidt et $\mathrm{al}^{10}$ implicated the activation of heparanase in degradation of glycocalyx in pulmonary circulatory beds. Based on the pathogenic role of circulating histone proteins in mediating acute lung injury, Freeman et $\mathrm{al}^{34}$ proposed that the high proportion of positively charged amino acids in histones could be involved in their electrostatic accumulation on the polyanionic surface of EG. Using tagged histones, they demonstrated their preferential and avid binding in rabbit lungs, which could be abolished by the administration of competing polycations or by heparanase, thus localizing the binding sites to the HS moieties of endothelial glycocalyx. Moreover, a number of HS analogues also prevented histone accumulation in the lungs by neutralizing circulating histones. Degradation of EG with the subsequent microcirculatory abnormalities explain, at least in part, the development of the ventilation-perfusion mismatch. Morphologic perturbations in pulmonary microvasculature were comprehensively studied by Inagawa et al. ${ }^{35}$ In endotoxemic mice, both scanning and transmission electron microscopy of EG, normally appearing as a mosslike structure, showed that it was severely disrupted, peeled away and coagulated, with numerous spherical structures, which were distinct from exosomes and contained elements of EG, appearing within capillary lumens.

\section{Cardiomyopathy}

Cardiomyopathy manifests in left ventricular dilatation, reduced cardiac contractility, and systolic or diastolic dysfunction with reduced response to volume expansion. ${ }^{36,37}$ TLRs expressed on cardiomyocytes respond to LPS and other pathogen-associated molecular patterns, such as bacterial wall lipoproteins, to activate NF- $\mathrm{kb}$ signaling, leading to the formation of proinflammatory cytokines. ${ }^{38}$ The resulting activation of sheddases leads to the degradation of glycocalyx and the release of HS fragments acting as highly potent damage-associated molecular patterns. Annecke et $\mathrm{al}^{11}$ attribute this step, present in ischemia reperfusion injury, to the activation of mast cells releasing the stored tryptase $\beta$, potentially acting as a sheddase. Similar mechanisms may be invoked in the development of cardiorenal syndrome type $\mathrm{V}-\mathrm{a}$ concomitant cardiac and renal dysfunction secondary to a systemic condition. It afflicts $40 \%$ to $60 \%$ of patients with sepsis. ${ }^{39}$

\section{Kidney Injury}

Kidney injury occurs in at least $40 \%$ patients with severe sepsis, and their combination is associated with a higher mortality rate. ${ }^{40}$ Some clinical features of sepsis, such as hypoalbuminemia, reduce effective circulating blood volume. Albuminuria and edema are attributed to the endotoxemia- and cytokine-induced increase in vascular endothelial permeability. ${ }^{1,41}$ The development of albuminuria is considered to be an indicator of damage to glomerular filtration barrier and perturbed uptake of filtered albumin by the tubular megalin and cubilin endocytic systems in the proximal tubule. ${ }^{42,43}$ A recent electron microscopy study of glomerular endothelia in septic mice ${ }^{44}$ revealed endotoxemia-induced precipitous drop in the density of fenestrae and a significant increase in the diameter of individual fenestrae. The total fenestrated area was found to be reduced to $12 \%$, compared to $23 \%$ in control mice. Endothelial fenestrae are normally covered by glycocalyx. Wheat germ agglutinin and HS antibody staining of EG 


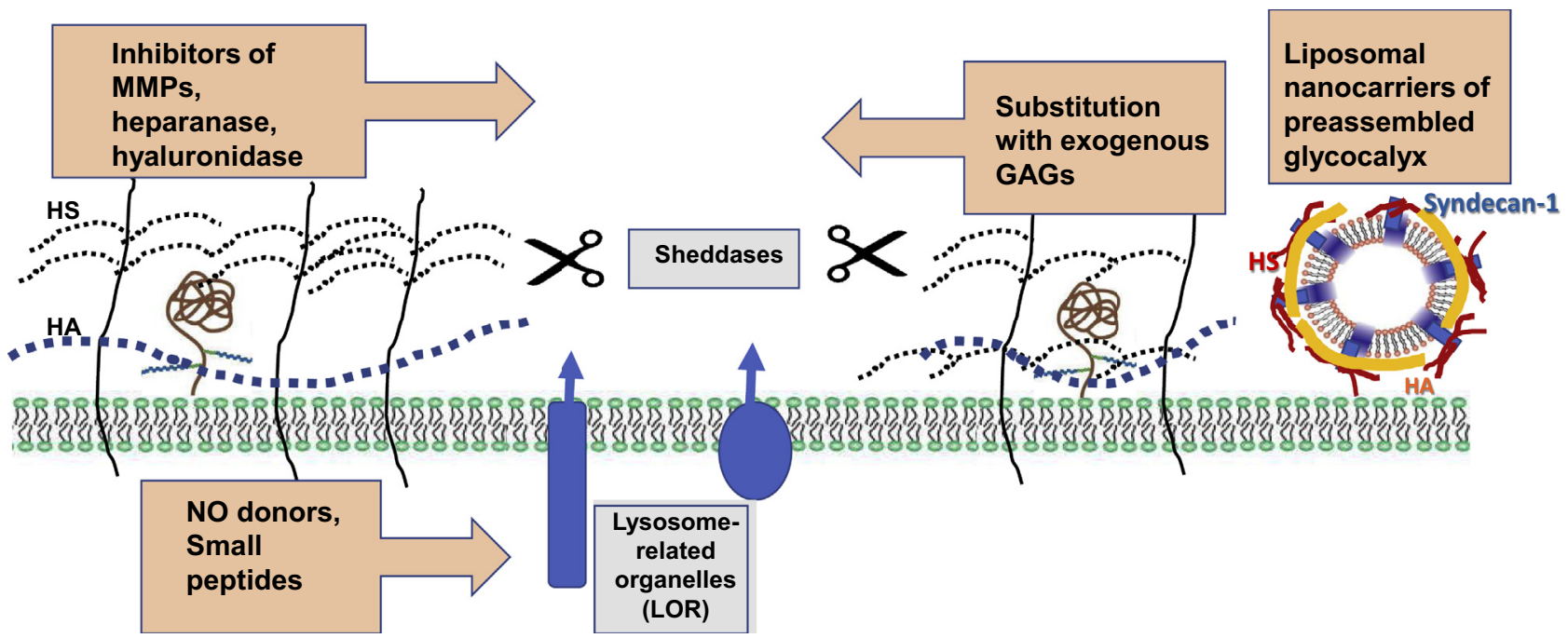

Figure 2 Groups of compounds proposed to prevent degradation and accelerate restoration of glycocalyx. Diverse families of proposed therapeutics are shown (orange boxed areas). Gray boxed areas reiterate mechanisms of glycocalyx degradation. The authors have recently proposed the use of liposomal nanocarriers of preassembled glycocalyx, which in the bloodstream fuse with the plasma membrane of endothelial and circulating cells to expeditiously restore their lost glycocalyx. GAG, glycosaminoglycan; HA, hyaluronic acid; HS, heparan sulfate; MMP, matrix metalloproteinase; NO, nitric oxide.

showed a substantial reduction in the staining pattern in glomeruli of endotoxemic mice with the concomitant increase in heparanase expression. These changes were absent in TNF receptor 1 knockout mice. These studies convincingly demonstrated $\mathrm{TNF} \alpha$-induced damage to the endothelial component of glomerular filtration barrier and implicated EG in these abnormalities.

\section{Therapeutic Approaches to Preventing Degradation and Accelerating Restoration of Glycocalyx}

Earlier failure of TLR4 inhibitors to ameliorate sepsis, ${ }^{2,3}$ the discovery of a noncanonical pathway of LPS-induced activation of inflammasome, ${ }^{4,5}$ and experimental documentation of the loss of glycocalyx in sepsis, ${ }^{10,13,14}$ together with the realization of an important role played by glycocalyx in microcirculatory homeostasis, ${ }^{15,19}$ have motivated an intense search for therapeutic modalities to prevent glycocalyx loss and to accelerate its restoration.

Natural restoration of EG is lengthy, requiring days to reconstitute functional EG. Attempts to accelerate EG restoration have been entertained. The administration of antioxidants, such as $\mathrm{N}$-acetylcysteine, ${ }^{45}$ was used for preventing EG shedding during hyperglycemia. Another strategy consisted of the systemic use of high-molecular-weight HA. A study by Henry and Duling ${ }^{46}$ demonstrated that supplemental infusion of HA and chondroitin sulfate accelerated restoration of EG. Doxycycline has been advocated for the prevention of glycocalyx degradation, reportedly due to an inhibitory effect on MMPs. ${ }^{47}$ Annecke et $\mathrm{al}^{48}$ examined the effects of volatile anesthetics on glycocalyx and demonstrated that sevoflurane exerts glycocalyx- protective qualities against ischemia reperfusion-induced degradation through its ability to attenuate the release of lysosomal cathepsin B.

In recent years, the ideas of glycocalyx restoration have percolated. One of the examples of this trend relates to the compound Corline heparin conjugate (Corline Biomedical, Uppsala, Sweden) and is based on preassembled aggregates of $>20$ heparin molecules using Corline heparin conjugate technology, which itself is protected by $>20$ granted or pending patents. This compound has apparent antithrombotic effects, but lacks the ability to mimic other functions of EG. Renaparin (Horizon 2020, project ID: 756195) is being tested for improving kidney transplantation outcomes and is reported to be used for generating antithrombogenic surface of islets of Langerhans. ${ }^{49}$ Nordling et $\mathrm{al}^{50}$ reported protection of the endothelium and improved early outcomes in kidney transplantation in pig and mouse models.

Wodicka et $\mathrm{al}^{51}$ developed a selectin-targeting antiadhesive coating (termed EC-SEAL) consisting of a dermatan sulfate backbone and multiple selectin-binding peptides specifically designed for binding to inflamed endothelium and preventing endothelium-platelet interaction.

\section{Heparin-Mimetic Compounds}

Heparin-mimetic compounds proposed to prevent and restore EG include the following members. Sulodexide, a mixture of HS and dermatan sulfate, has properties of both glycosaminoglycans and inhibitors of sheddases. Song et $\mathrm{a}^{14}$ demonstrated that sulodexide is capable of preventing glycocalyx degradation and improving survival in mice with polymicrobial sepsis. Rhamnan sulfate is another 
heparin-mimetic compound capable of improving HS cell coat and vascular permeability. ${ }^{52}$

\section{S1P/Albumin-S1P}

S1P/albumin-S1P has been associated with improved glycocalyx permeability, probably related to the S1P-induced inhibition of sheddases. ${ }^{53}$

\section{Heparanase Inhibitors}

Heparanase inhibitors are emerging, the subject discussed in depth elsewhere $e^{10,25,26,54}$ and in this issue.

\section{Cationic Copolymers}

Cationic copolymers such as methacrylamidopropyltrimethyl ammonium chloride-based copolymers have been reported to improve endothelial barrier function. ${ }^{55}$ Holland et $\mathrm{al}^{56}$ proposed a biomimetic surface modification of graphite using oligosaccharide surfactant polymers consisting of a flexible poly (vinyl amine) with dextran and alkanoyl side chains. This structure, according to the investigators, creates a glycocalyx-like coating. The idea of using polymers to protect glycocalyx has found other proponents demonstrating the effects of high-molecularweight polyethylene glycol in human lung endothelial cell barrier regulation $^{57}$ or in the form of polyethylene glycol-NO compound shown to normalize arteriolar response and oxidative stress in ischemia reperfusion. ${ }^{58}$ Another modality shown to prevent EG degradation in endotoxemia is the administration of antithrombin. ${ }^{59}$

\section{Reconditioning Therapy}

In many instances, especially in sepsis, a speedy restoration of EG may be decisive the in life-or-death fate of patients. In view of this requirement, a fast restoration of glycocalyx was sought, and in this vein, a strategy for reconditioning therapy for glycocalyx was developed. Therefore, an attempt was made to mimic glycocalyx by injecting liposomal nanocarriers of preassembled glycocalyx (LNPGs; patent publication number US2019/0091257 A1, March 28, 2019) into the circulation, as previously described. ${ }^{18}$ Based on the negative charge of LNPGs, they adhere with at least sixfold higher affinity to endothelial cells with degraded glycocalyx (Figure 2). In proof-of-concept studies in mice injected with the lethal dose of LPS, the administration of LNPGs 1 hour later was associated with significantly improved survival (M.S.G. and D.S., unpublished data). Blood pressure telemetry in these mice showed that the administration of LNPGs significantly improved blood pressure control and myocardial contractility, and reduced vascular permeability (D.S., unpublished data). We hypothesize that the observed beneficial effects of LNPGs could be attributed to their fusion with the plasma membrane, predominantly at the sites where glycocalyx has been degraded, and exposing on the surface HS proteoglycans, such as syndecan- 1 .

\section{Conclusions and Perspectives}

This brief overview integrates the lines of evidence on the role played by glycocalyx in sepsis and advances ideas of glycocalyx preservation and restoration as rational strategies in mitigating manifestations of sepsis. Of course, it would be overly simplistic to regale the multifaceted pathogenesis of sepsis to the loss of glycocalyx integrity. Yet, a score of pathophysiological manifestations of sepsis is attributable, at least in part, to this singular and chronologically early event: the degradation of glycocalyx. By interfering with all steps of leukocyte-endothelial interaction, it hinders bacterial clearance. By reducing the level of glycocalyx-bound antithrombin III, it predisposes to thrombosis. By failing to effectively transduce mechanical forces to endothelial NO synthase, it bestows stagnation of the blood flow and imparts platelet aggregation. By the loss of high-molecularweight hyaluronan, it increases vascular permeability. By truncating HS moieties, it reduces the concentration of growth and survival factors and extracellular antioxidant defense in the outer layer of the cells and predisposes to their apoptosis. And by generating excessive amounts of circulating fragments activating TLRs, it invigorates the innate immune response. These sequelae of glycocalyx degradation are paramount for circulatory collapse and the development of septic shock. Hence, future strategies and pharmacologic tools for preventing degradation and restoring the integrity of glycocalyx should be prioritized.

\section{References}

1. Aird W: The role of the endothelium in severe sepsis and multiorgan dysfunction syndrome. Blood 2003, 101:3765-3777

2. Rice TW, Wheeler AP, Bernard GR, Vincent JL, Angus DC Aikawa N, Demeyer I, Sainati S, Amlot N, Cao C, Ii M, Matsuda H, Mouri K, Cohen J: A randomized, double-blind, placebo-controlled trial of TAK-242 for the treatment of severe sepsis. Crit Care Med 2010, 38:1685-1694

3. Laterre PF, Francois B, LaRosa SP, Angus DC, Mira JP, Wittebole X, Dugernier T, Perrotin D, Tidswell M, Jauregui L, Krell K, Pachl J, Takahashi T, Peckelsen C, Cordasco E, Chang CS, Oeyen S, Aikawa N, Maruyama T, Schein R, Kalil AC, Van Nuffelen M, Lynn M, Rossignol DP, Gogate J, Roberts MB, Wheeler JL, Vincent JL; ACCESS Study Group: Effect of eritoran, an antagonist of MD2-TLR4, on mortality in patients with severe sepsis: the ACCESS randomized trial. JAMA 2013, 309:1154-1162

4. Kayagaki N, Warming S, Lamkanfi M, Vande Walle L, Louie S, Dong J, Newton K, Qu Y, Liu J, Heldens S, Zhang J, Lee WP, RooseGirma M, Dixit VM: Non-canonical inflammasome activation targets caspase-11. Nature 2011, 479:117-121

5. Shi J, Zhao Y, Wang Y, Gao W, Ding J, Li P, Hu L, Shao F: Inflammatory caspases are innate immune receptors for intracellular LPS. Nature 2014, 514:187-192

6. Huet O, Chin-Dusting J: Septic shock: desperately seeking treatment. Clin Sci 2014, 128:31-39 
7. Hawiger I, Veach R, Zienkiewicz J: New paradigms in sepsis: from prevention to protection of failing microcirculation. J Thromb Haemost 2015, 13:1743-1756

8. Morrison D, Ulevitch R: The effect of bacterial endotoxins on host mediation systems: a review. Am J Pathol 1978, 93:526-617

9. Becker B, Chappell D, Jacob M: Endothelial glycocalyx and coronary vascular permeability: the fringe benefit. Basic Res Cardiol 2010, 105:687-701

10. Schmidt E, Yang Y, Janssen W, Gandjeva A, Perez MJ, Barthel L, Zemans RL, Bowman JC, Koyanagi DE, Yunt ZX, Smith LP, Cheng SS, Overdier KH, Thompson KR, Geraci MW, Douglas IS, Pearse DB, Tuder RM: The pulmonary endothelial glycocalyx regulates neutrophil adhesion and lung injury during experimental sepsis. Nat Med 2012, 18:1217-1223

11. Annecke T, Fischer J, Hartmann H, Tschoep J, Rehm M, Conzen P, Sommerhoff CP, Becker BF: Shedding of the coronary endothelial glycocalyx: effects of hypoxia/reoxygenation vs ischemia/reperfusion. Br J Anaesth 2011, 107:679-686

12. Mulivor A, Lipowsky H: Inflammation and ischemia-induced shedding of venular glycocalyx. Am J Physiol Heart Circ Physiol 2004, 286:H1672-H1680

13. Zullo J, Fan J, Azar T, Yen W, Zeng M, Chen J, Ratliff BB, Song J, Tarbell JM, Goligorsky MS, Fu BM: Exocytosis of endothelial lysosome-related organelles hair-triggers a patchy loss of glycocalyx at the onset of sepsis. Am J Pathol 2016, 186:248-258

14. Song JW, Zullo JA, Liveris D, Dragovich M, Zhang XF, Goligorsky MS: Therapeutic restoration of endothelial glycocalyx in sepsis. J Pharmacol Exp Ther 2017, 361:115-121

15. Reitsma S, oude Egbrink M, Vink H, van den Berg B, Pasos V, Engels W, Slaaf D, van Zandvoort M: Endothelial glycocalyx structure in the internal carotid artery: a two-photon laser scanning microscopy study. J Vasc Res 2011, 48:297-306

16. Yen W, Cai B, Zeng M, Tarbell J, Fu B: Quantification of the endothelial surface glycocalyx on rat and mouse blood vessels. Microvasc Res 2012, 83:337-346

17. Levi M, van der Poll T: Endothelial injury in sepsis. Intensive Care Med 2013, 39:1839-1842

18. Zhang X, Sun D, Song J, Zullo J, Lipphardt M, Cohen-Gould L, Goligorsky MS: Endothelial cell dysfunction and glycocalyx - a vicious circle. Matrix Biol 2018, 71-72:421-431

19. Wyngene L, Wandewalle J, Libert C: Reprogramming of basic metabolic pathways in microbial sepsis: therapeutic targets at last? EMBO Mol Med 2018, 10:e8712

20. McGarrity S, Anuforo O, Halldorsson H, Bergmann A, Halldorsson S, Palsson S, Henriksen H, Johansson P, Rolfsson O: Metabolic systems analysis of LPS-induced endothelial dysfunction applied to septic patient stratification. Sci Rep 2018, 8:6811

21. Mishra H, Ma J, Walcheck B: Ectodomain shedding by ADAM17: its role in neutrophil recruitment and the impairment of this process during sepsis. Front Cell Infect Microbiol 2017, 7:138

22. Lin J, Chan O, Hsiao H, Wang Y, Hsia S, Chiu C: Decreased ADAM13 activity is associated with disease severity and outcome in pediatric severe sepsis. Medicine (Baltimore) 2016, 95:e3374

23. Levin M, Udi Y, Solomonov I, Sagi I: Next generation MMP inhibitors - novel strategies bring new prospects. Biochem Biophys Acta Mol Cell Res 2017, 1864:1927-1939

24. Vanlaere I, Libert C: MMPs as drug targets in infections caused by gram-negative bacteria and septic shock. Clin Microbiol Rev 2009, 22:224-239

25. Goldberg R, Meirovitz A, Hirshoren N, Bulvik R, Binder A, Rubinstein A, Elkin M: Versatile role of heparanase in inflammation. Matrix Biol 2013, 32:234-240

26. Ilan N, Elkin M, Vlodavsky I: Regulation, function and clinical significance of heparanase in cancer metastasis and angiogenesis. Int J Biochem Cell Biol 2006, 38:2018-2039
27. Powell J, Horton M: Threat matrix: low-molecular-weight hyaluronan as a danger signal. Immunol Res 2005, 31:207-218

28. Stern R, Asari A, Sugahara K: Hyaluronan fragments: an informationrich system. Eur J Cell Biol 2006, 85:699-715

29. Nelson A, Berkestedt I, Bodelsson M: Circulating glycosaminoglycan species in septic shock. Acta Anaesthesiol Scand 2014, 38:36-43

30. Decleves A, Caron N, Voisin V, Legrand A, Bouby N, Kultti A, Tammi M, Flamion B: Synthesis and fragmentation of hyaluronan in renal ischemia. Nephrol Dial Transplant 2012, 27:3771-3781

31. Wilke S, Krausze J, Bussow K: Crystal structure of the conserved domain of the DC lysosomal associated membrane protein: implications for the lysosomal glycocalyx. BMC Biol 2012, 10:62

32. Settembre C, Fraldi A, Medina D, Ballabio A: Signals from the lysosome: a control centre for cellular clearance and energy metabolism. Mol Cell Biol 2013, 14:283-296

33. Kuo MC, Patschan D, Patschan S, Cohen-Gould L, Park HC, Ni J, Addabbo F, Goligorsky MS: Ischemia-induced exocytosis of WeibelPalade bodies mobilizes stem cells. J Am Soc Nephrol 2008, 19: $2321-2330$

34. Freeman C, Parish C, Knox K, Blackmore J, Lobov S, King D, Senden T, Stephens R: The accumulation of circulating histones on heparan sulfate in the capillary glycocalyx of the lungs. Biomaterials 2013, 34:5670-5676

35. Inagawa R, Okada H, Takemura G, Suzuki K, Takada C, Yano H, Ando Y, Usui T, Hotta Y, Miyazaki N, Tsujimoto A, Zaikokuji R, Matsumoto A, Kawaguchi T, Doi T, Yoshida T, Yoshida S, Kumada K, Ushikoshi H, Toyoda I, Ogura S: Ultrastructural alteration of pulmonary capillary endothelial glycocalyx during endotoxemia. Chest 2018, 154:317-325

36. Beesley S, Weber G, Sarge T, Nikravan S, Grissom CK, Lanspa MJ, Shahul S, Brown SM: Septic cardiomyopathy. Crit Care Med 2018, 46:625-634

37. Martin L, Derwall M, Al Zoubi S, Zechendorf E, Reuter D, Thiemermann C, Schuerholz T: The septic heart: current understanding of molecular mechanisms and clinical implications. Chest 2019, 155:427-437

38. Takeuchi O, Akira S: Pattern recognition receptors and inflammation. Cell 2010, 140:805-820

39. Kotecha A, Vallabhajosyula S, Coville HH, Kashani K: Cardiorenal syndrome in sepsis: a narrative review. J Crit Care 2018, 43:122-127

40. Oppert M, Engel C, Brunkhorst F, Bogatsch H, Reinhart K, Frei U, Eckardt KU, Loeffler M, John S; German Competence Network Sepsis (Sepnet): Acute renal failure in patients with severe sepsis and septic shock - a significant independent risk factor for mortality: results from the German prevalence study. Nephrol Dial Transplant 2008, 23:904-909

41. Cunningham P, Dyanov H, Park P, Wang J, Newell KA, Quigg RJ: Acute renal failure in endotoxemia is caused by TNF acting directly on TNF receptor-1 in kidney. J Immunol 2002, 168:5817-5823

42. De Gaudio A, Adembri C, Grechi S, Novelli G: Microalbuminuria as an early index of impairment of glomerular permeability in postoperative septic patients. Intensive Care Med 2000, 26:1364-1368

43. Schreiber A, Theilig F, Schweda F, Höcherl K: Acute endotoxemia in mice induces downregulation of megalin and cubilin in the kidney. Kidney Int 2012, 82:53-59

44. Xu C, Chang A, Hack B, Eadon M, Alper S, Cunningham P: TNFmediated damage to glomerular endothelium is an important determinant of acute kidney injury in sepsis. Kidney Int 2013, 85:72-81

45. van den Berg B, Nieuwdrop M, Stroes E, Vink H: Glycocalyx and endothelial (dys)function: from mice to men. Pharmacol Rep 2006, 58 Suppl:75-80

46. Henry C, Duling B: Permeation of the luminal capillary glycocalyx is determined by hyaluronan. Am J Physiol 1999, 277:H508-H514

47. Lipowsky HH, Lescanic A: The effect of doxycycline on shedding of the glycocalyx due to reactive oxygen species. Microvasc Res 2013, 90:80-85 
48. Annecke T, Chappell D, Chen C, Jacob M, Welsch U, Sommerhoff C, Rehm M, Conzen P, Becker B: Sevoflurane preserves the endothelial glycocalyx against ischaemia-reperfusion injury. Br J Anaesth 2010, 104:414-421

49. Cabric S, Torsten E, Sanchez J, Nilsson B, Korsgren O, Larsson R: A new method for incorporating functional heparin onto the surface of islets of Langerhans. Tissue Eng Part C Methods 2008, 14: $141-147$

50. Nordling S, Brannstrom J, Carlsson F, Lu B, Salvaris E, Wanders A, Buijs J, Estrada S, Tolmachev V, Cowan P, Lorant T, Magnusson P: Enhanced protection of the renal vascular endothelium improves early outcomes in kidney transplantation: preclinical investigations in pig and mouse. Sci Rep 2018, 8:5220

51. Wodicka J, Chambers A, Sangha G, Goergen C, Panitch A: Development of a glycosaminoglycan derived, selectin targeting antiadhesive coating to treat endothelial cell dysfunction. Pharmaceuticals (Basel) 2017, 10:36

52. Cancel L, Tarbell J: Rhamnan sulfate enhances the endothelial glycocalyx and decrease the LDL permeability of human coronary artery endothelial cells in vitro. FASEB J 2013, 27:896.3

53. Tarbell J, Cancel L: The glycocalyx and its significance in human medicine. J Intern Med 2016, 280:97-113
54. Vlodavsky I, Singh P, Boyango I, Gutter-Kapon L, Elkin M, Sanderson R, Ilan N: Heparanase: from basic research to therapeutic applications in cancer and inflammation. Drug Resist Updat 2016, 29: $54-75$

55. Giantsos-Adams K, Lopez-Quintero V, Kopeckova P, Kopecek J, Tarbell J, Dull R: Study of the therapeutic benefit of cationic copolymer administration to vascular endothelium under mechanical stress. Biomaterials 2011, 32:288-294

56. Holland N, Qui Y, Ruegsegger M, Marchant R: Biomimetic engineering of non-adhesive glycocalyx-like surfaces using oligosaccharide surfactant polymers. Nature 1998, 392:799-801

57. Chiang E, Camp SM, Dudek SM, Brown ME, Usatyuk PV Zaborina O, Alverdy JC, Garcia JG: Protective effects of highmolecular weight polyethylene glycol (PEG) in human lung endothelial cell barrier regulation: role of actin cytoskeletal rearrangement. Microvasc Res 2009, 77:174-186

58. Bertuglia S, Veronese FM, Pasut G: Polyethylene glycol and a novel developed polyethylene glycol-nitric oxide normalize arteriolar response and oxidative stress in ischemia-reperfusion. Am J Physiol Heart 2006, 291:H1536-H1544

59. Iba T, Levy J, Hirota T, Hiki M, Sato K, Murakami T, Nagaoka I: Protection of the endothelial glycocalyx by antithrombin in an endotoxin-induced rat model of sepsis. Thromb Res 2018, 171:1-6 